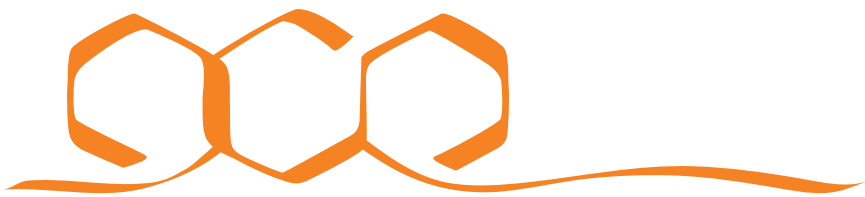 COMMUNICATIONS CHEMISTRY
}

COMMENT

https://doi.org/10.1038/s42004-020-00419-5 OPEN

\section{Open questions on aromaticity in organometallics}

\author{
Jun Zhu (iD ${ }^{1 \times}$
}

While $s p^{2}$-hybridized carbon atoms in hydrocarbons typically contribute only one electron to their aromaticity, metals have more electrons from $d$ or $f$ orbitals available for participating in conjugation in organometallics, complicating the electron counting as well as analysis of their aromaticity. Here, the author comments on the challenges towards understanding aromaticity in organometallics and outlines several remaining questions that have yet to be answered.

The concept of aromaticity, originally proposed for benzene and hydrocarbons with low C:H ratios owing to their pleasant smells (aromas), was used to explain their enhanced thermodynamic stabilities in comparison with their acyclic counterparts, caused by their resonance structures (Fig. 1a) $)^{1,2}$. Nowadays, aromaticity has been extended from organics to organometallics $^{3}$ to inorganics ${ }^{4}$, and from the ground state to the excited state 5,6 , leading to various applications of aromatics in functional materials and reaction mechanisms ${ }^{7,8}$. In comparison with traditional organics, analyzing the aromaticity in organometallics (Fig. 1) is still challenging and several open questions are listed below to invite both experimentalists and theoreticians for their examination.

\section{The nature of aromaticity in organometallics}

Although various aromaticity indices have been proposed, it is still challenging to quantify aromaticity in organometallics. For instance, nucleus-independent chemical shift (NICS) values could be significantly affected by the metal centers ${ }^{9}$ or ligands close to a ghost atom (placed at the ring center for a $\sigma$ system or $1.0 \AA$ above or below the ring center for a $\pi$ system in most cases). The harmonic oscillator model of aromaticity (HOMA) values are also unavailable in organometallics due to a lack of a parameterization for transition metal-carbon bonds ${ }^{10}$. Whatever method is used, trying aromaticity indices (based on different physicochemical properties) as much as possible is always recommended ${ }^{11}$. In many cases, the contradictions between different indices are caused by the failure of some indicators to evaluate aromaticity correctly rather than by the multidimensional character of aromaticity. On the other hand, the current classification of aromaticity in organometallics is mainly based on the topology and

\footnotetext{
${ }^{1}$ State Key Laboratory of Physical Chemistry of Solid Surfaces and Collaborative Innovation Center of Chemistry for Energy Materials (iChEM), Fujian Provincial Key Laboratory of Theoretical and Computational Chemistry and Department of Chemistry, College of Chemistry and Chemical Engineering, Xiamen University, Xiamen 361005, China. ${ }^{凶}$ email: jun.zhu@xmu.edu.cn
} 
number of $\pi$-electrons, similar to that in organics, leading to two types of aromaticity, specifically, Hückel aromaticity (with $4 n$ $+2 \pi$-electrons $)^{12}$ and Möbius aromaticity $(4 n \pi \text {-electrons })^{13}$. However, early in 1958, 6 years before Heilbronner proposed the concept of Möbius aromaticity ${ }^{13}$, Craig et al. proposed a novel type of aromaticity ${ }^{14}$, which was later termed as Craig-type Möbius aromaticity ${ }^{15}$. Note that when more rings are involved in the conjugation, exclusively classifying some metallacycles as having Hückel or Möbius aromaticity becomes difficult and even unnecessary because they have a hybrid Hückel-Möbius or even quasi-aromatic nature ${ }^{16}$. To give a full credit to Craig, the original proposer for $4 n \pi$-aromaticity, an alternative is proposed here that three rather than two types of aromaticity in organometallics could be more suitable, namely, Hückel aromaticity, Craig aromaticity and Möbius aromaticity (Fig. 1b). The first one contains $4 n+2 \pi$-electrons, whereas both the second and the third contain $4 n$ effective $\pi$-electrons. The difference between the second and the third solely depends on the topology of a molecule. The former has a planar ring whereas the latter possesses a Möbius topology.

\section{Electron counting on hyperconjugative aromaticity in organometallics}

The electronic configuration (ground state) of carbon is $1 s^{2} 2 s^{2} 2 p^{2}$, whereas that of transition metals becomes $(n-1)$ $d^{1: 10} n s^{1: 2}(n=4,5,6)$. Thus, more electrons from $d$ orbitals of a transition metal, in contrast to one electron from a carbon atom, can participate in the conjugation of a cyclic species, leading to a challenge in electron counting in organometallics. For instance, the number of $\pi$-electrons in a metallabenzene complex could vary from six to ten depending on metal centers, geometries and ligands ${ }^{17}$. In addition, the number of formal $\pi$-electrons of the first isolated metallapentalyne is ten. However, the nature of its aromaticity is eight-center-eight-electron Craig aromaticity ${ }^{18}$. Thus, electron counting in metallacycles is far from trivial. It is particularly challenging to determine the number of $\pi$-electrons in hyperconjugative aromaticity of organometallics as more $\pi$ electrons than six could be located in transition metal-substituted pyrrolium rings ${ }^{19}$.

\section{$\sigma$-aromaticity in unsaturated organometallics}

In general, aromaticity could be classified as $\sigma$ - and $\pi$-aromaticity according to the character of the cyclic electron delocalization, distributed in saturated and unsaturated systems, respectively. Crossing to the opponent's domain seems unlikely. Interestingly, $\sigma$-aromaticity dominating in an unsaturated organometallic system has been reported recently in a metallacyclopropene unit ${ }^{3}$.
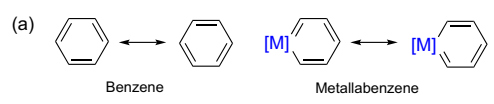

(b)
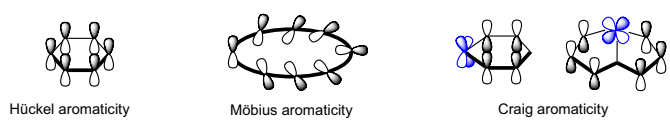

(c)
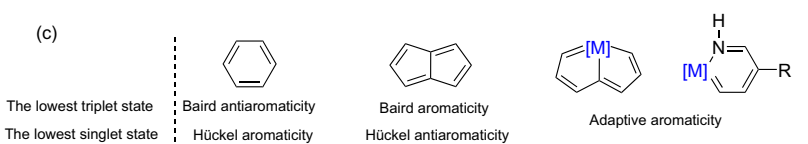

Fig. 1 Comparison of aromaticity between organics and organometallics in both the lowest singlet and triplet states. a The resonance structures of benzene, metallabenzene, and metallapentalene. b Proposed alternative classification of aromaticity. c Adaptive aromaticity in organometallics in comparison with traditional Hückel and Baird aromaticity. [M] = A metal fragment; $R=A$ phosphonium substituent.
It is still challenging to dig out $\sigma$-aromatic unsaturated organometallics, especially in other-membered rings because $\pi$-aromaticity in most cases prevails in such an unsaturated domain.

\section{Adaptive aromaticity in organometallics}

Cyclic species can be aromatic either in the ground state or the excited state according to Hückel and Baird rules, which could be regarded as one-state aromaticity. Aromatic species that exist in two states, e.g., the lowest singlet state $\left(\mathrm{S}_{0}\right)$ and the lowest triplet state $\left(T_{1}\right)$ seems impossible owing to a violation of Hückel and Baird rules. Very recently, our group proposed two-state aromaticity in a 16e osmapentalene (Fig. 1c), which is aromatic in both $\mathrm{S}_{0}$ and $\mathrm{T}_{1}$ states, and which we termed as adaptive aromaticity $^{20}$. Later, the concept of adaptive aromaticity has been extended to osmapyridinium ${ }^{21}$, mono-substituted benzene, tetraatomic boron species, osmapentalene derivatives, cyclo[10] carbon, and the pyrrole ring in dipyrrolonaphthyridinedione ${ }^{22}$. As two-state aromaticity is particularly rare, discovery of such adaptive aromatics, caused by a novel excitation fashion, has been always challenging. As the nature of aromaticity in metallabenzene remains ambiguous, the unpredictability in adaptive aromaticity could become more significant.

\section{Aromaticity-driven dinitrogen activation in organometallics}

Aromaticity could stabilize not only an intermediate or a product in a given reaction but also the transition state. Recently, we proposed a novel approach for dinitrogen activation by a frustrated Lewis pair $\mathbf{1}$ via density functional theory calculations (Fig. 2) 23 . The number of aromatic rings could be gradually increased from one (in a reactant) to two (in a transition state) to three (in a product) via a $[4+2]$ cycloaddition, which is supported by the negative $\operatorname{NICS}(1)_{z z}$ values and clockwise ring currents in the anisotropy of the current-induced density (ACID) plots. Thus, dinitrogen activation becomes favorable both thermodynamically (with an exothermicity of Gibbs free energy by $18.9 \mathrm{kcal} \mathrm{mol}^{-1}$ ) and kinetically (with an activation energy as low as $9.1 \mathrm{kcal} \mathrm{mol}^{-1}$ ). Developing aromaticity-driven transition meal-involved dinitrogen activation should be challenging due to the strong triple bond of dinitrogen, but represents an example where aromaticity in organometallics could potentially be harnessed for practical applications.

\section{Outlook}

The diversity of transition metals significantly enriches the chemistry of metalla-aromatics where a metal fragment is used to replace the $\mathrm{CH}$ group in an organic species. In particular,

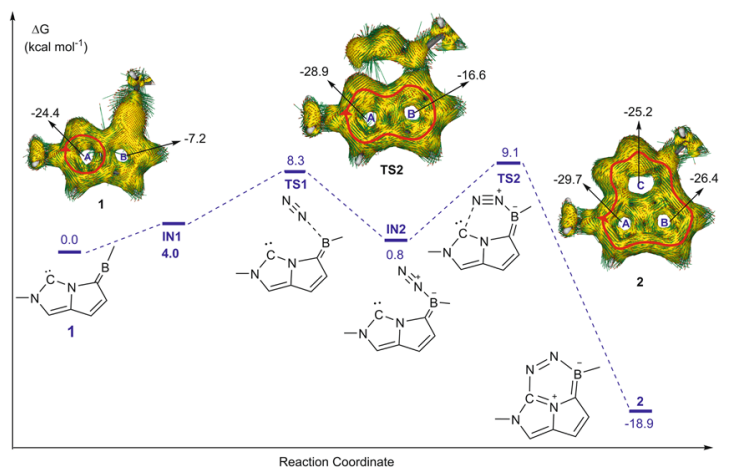

Fig. 2 Predicted dinitrogen activation by a frustrated Lewis pair. Gibbs free energy profiles for the activation of $\mathrm{N}_{2}$ by carbon-boron frustrated Lewis pair $\mathbf{1}$ with selected ACID plots with an isovalue of 0.04 a.u. and $\operatorname{NICS}(1)_{z z}$ values (ppm) of rings $A, B$, and $C$ in $\mathbf{1}, \mathbf{T S 2}$, and $\mathbf{2}$. Adapted with permission from ref. ${ }^{23}$. Copyright 2019 Wiley-VCH Verlag GmbH \& Co. 
metal-bridgehead aromatics and spiro metalla-aromatics become possible as some of the organic counterparts are either particularly challenging or impossible due the limited mode of the carbon coordination ${ }^{18,24}$. As every coin has two sides, the more electrons from $d$ or $f$ orbitals definitely complicate the aromaticity in organometallics, enhancing the uncertainties of aromaticity. For instance, the aromaticity reversal between metalla-aromatics and the organic parent has been found in carbolong chemistry from Hückel aromaticity to Craig aromaticity ${ }^{8}$. On the other hand, in comparison with various metalla-aromatics, realizing metalla-antiaromatics is even more challenging owing to the destabilization of antiaromaticity. As most reported organometallics are transition metal involved, developing $f$-block aromatic organometallics ${ }^{25}$ is extremely challenging as most $f$-block elements favor a bonding with oxygen and nitrogen atoms. With the rapid development of computational power, calculations will not only contribute significantly to understanding the aromaticity of transition states as well as products in reaction mechanisms in organometallic chemistry, but also play an important role in predicting novel metalla-(anti)aromatics and their computationally screened pathways for experimental examination.

Received: 29 September 2020; Accepted: 14 October 2020;

Published online: 10 November 2020

\section{References}

1. Schleyer, P. V. R. Introduction: delocalization Pi and Sigma. Chem. Rev. 105, 3433-3435 (2005).

2. Gleiter, R. \& Haberhauer, G. Aromaticity and other conjugation effects. (Wiley-VCH: Weinheim, 2012).

3. Chen, D., Xie, Q. \& Zhu, J. Unconventional aromaticity in organometallics: the power of transition metals. Acc. Chem. Res. 52, 1449-1460 (2019).

4. Boldyrev, A. I. \& Wang, L.-S. All-metal aromaticity and antiaromaticity. Chem. Rev. 105, 3716-3757 (2005).

5. Baird, N. C. Quantum organic photochemistry. II. Resonance aromaticity lowest ${ }^{3} \pi \pi^{*}$ state cycl. hydrocarbons. J. Am. Chem. Soc. 94, 4941-4948 (1972).

6. Rosenberg, M., Dahlstrand, C., Kilså, K. \& Ottosson, H. Excited state aromaticity and antiaromaticity: opportunities for photophysical and photochemical rationalizations. Chem. Rev. 114, 5379-5425 (2014).

7. Chen, S. et al. Addition of alkynes and osmium carbynes towards functionalized $d_{\pi}-p_{\pi}$ conjugated systems. Nat. Commun. 11, 4651 (2020)

8. Chen, D., Hua, Y. \& Xia, H. Metallaaromatic chemistry: history and development. Chem. Rev. 120, https://doi.org/10.1021/acs.chemrev.0c00392 (2020).

9. Nejad, C. F., Vícha, J. \& Ghosh, A. Relativity or aromaticity? A first-principles perspective of chemical shifts in osmabenzene and osmapentalene derivatives. Phys. Chem. Chem. Phys. 22, 10863-10869 (2020).

10. Krygowski, T. M., Szatylowicz, H., Stasyuk, O. A., Dominikowska, J. \& Palusiak, M. Aromaticity from the viewpoint of molecular geometry: application to planar systems. Chem. Rev. 114, 6383-6422 (2014).

11. Solà, M. Why aromaticity is a suspicious concept? why? Front. Chem. 5, 22 (2017).

12. Hückel, E. Quantentheoretische Beiträge zum Benzolproblem. Z. Phys. 70, 204-286 (1931).

13. Heilbronner, E. Hückel molecular orbitals of Möbius-type conformations of annulenes. Tetrahedron Lett. 5, 1923-1928 (1964).
14. Craig, D. P. \& Paddock, N. L. A novel type of aromaticity. Nature 181, 1052-1053 (1958)

15. Rzepa, H. S. Möbius aromaticity and delocalization. Chem. Rev. 105, 3697-3715 (2005).

16. Szczepanik, D. W. \& Solà, M. Electron delocalization in planar metallacycles: Hückel or Möbius aromatic? ChemistryOpen 8, 219-227 (2019).

17. Fernandez, I. \& Frenking, G. Aromaticity in metallabenzenes. Chem. Eur. J. 13, 5873-5884 (2007).

18. Zhu, C. et al. Stabilization of anti-aromatic and strained five-membered rings with a transition metal. Nat. Chem. 5, 698-703 (2013).

19. Sun, T., Xie, Q., Zhao, L. \& Zhu, J. Probing the most aromatic and antiaromatic pyrrolium rings by maximizing hyperconjugation and push-pull effect. Chem. Asian J. 13, 1419-1423 (2018).

20. Chen, D., Shen, T., An, K. \& Zhu, J. Adaptive aromaticity in $\mathrm{S}_{0}$ and $\mathrm{T}_{1}$ states of pentalene incorporating 16 valence electron osmium. Commun. Chem. 1, 18 (2018).

21. Shen, T., Chen, D., Lin, L. \& Zhu, J. Dual aromaticity in both the $\mathrm{T}_{0}$ and $\mathrm{S}_{1}$ states: osmapyridinium with phosphonium substituents. J. Am. Chem. Soc. 141, 5720-5727 (2019).

22. Huang, Y., Dai, C. \& Zhu, J. Adaptive $\sigma$-aromaticity in an unsaturated threemembered ring. Chem. Asian J. 15, https://doi.org/10.1002/asia.202000900 (2020).

23. Zhu, J. Rational design of a carbon-boron frustrated lewis pair for metal-free dinitrogen activation. Chem. Asian J. 14, 1413-1417 (2019).

24. Zhang, Y. L. et al. Spiro metalla-aromatics of Pd, Pt, and Rh: synthesis and characterization. J. Am. Chem. Soc. 139, 5039-5042 (2017).

25. Tsipis, A. C., Kefalidis, C. E. \& Tsipis, C. A. The role of the $5 \mathrm{f}$ orbitals in bonding, aromaticity, and reactivity of planar isocyclic and heterocyclic uranium clusters. J. Am. Chem. Soc. 130, 9144-9155 (2008).

\section{Acknowledgements}

Financial support by the Ministry of Education of China (H20200504), the National Science Foundation of China (22073079 and 21873079), and the Top-Notch Young Talents Program of China is gratefully acknowledged.

\section{Competing interests}

The author declares no competing interests.

\section{Additional information}

Correspondence and requests for materials should be addressed to J.Z.

Reprints and permission information is available at http://www.nature.com/reprints

Publisher's note Springer Nature remains neutral with regard to jurisdictional claims in published maps and institutional affiliations.

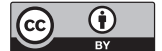

Open Access This article is licensed under a Creative Commons Attribution 4.0 International License, which permits use, sharing, adaptation, distribution and reproduction in any medium or format, as long as you give appropriate credit to the original author(s) and the source, provide a link to the Creative Commons license, and indicate if changes were made. The images or other third party material in this article are included in the article's Creative Commons license, unless indicated otherwise in a credit line to the material. If material is not included in the article's Creative Commons license and your intended use is not permitted by statutory regulation or exceeds the permitted use, you will need to obtain permission directly from the copyright holder. To view a copy of this license, visit http://creativecommons.org/ licenses/by/4.0/.

(C) The Author(s) 2020 